\title{
Suzaku observation of the black hole transient 4U1630-472: discovery of absorption lines
}

Aya Kubota, ${ }^{1}$ Tadayasu Dotani, ${ }^{2}$ Jean Cottam, ${ }^{3}$ Taro Kotani, ${ }^{4}$ Chris Done, ${ }^{2,5}$ Yoshihiro Ueda, ${ }^{6}$ Andy C. Fabian, ${ }^{7}$ Tomonori Yasuda, ${ }^{8}$ Hiromitsu Takahashi, ${ }^{8}$ Yasushi Fukazawa, ${ }^{8}$ Kazutaka Yamaoka, ${ }^{9}$ Kazuo Makishima, ${ }^{1,10}$ Shinya Yamada, ${ }^{10}$ Takayoshi Kohmura, ${ }^{11}$ Lorella Angelini ${ }^{3}$ and the Suzaku team

\footnotetext{
${ }^{1}$ Inst. of Physical and Chemical Research (RIKEN), 2-1 Hirosawa, Wako, Saitama 351-0198

${ }^{2}$ Institute of Space and Astronautical Science, Japan Aerospace Exploration Agency, 3-1-1 Yoshinodai, Sagamihara, Kanagawa 229-8510

${ }^{3}$ Exploration of the Universe Div., NASA GSFC, Greenbelt, MD 20771, USA

${ }^{4}$ Department of Physics, Tokyo Tech, 2-12-1 O-okayama, Meguro, Tokyo 152-8551, Japan

${ }^{5}$ Department of Physics, University of Durham, South Road, Durham, DH1 3LE, UK

${ }^{6}$ Department of Astronomy, Kyoto University, Sakyo-ku, Kyoto 606-8502, Japan

${ }^{7}$ Institute of Astronomy, Madingley Road, Cambridge CB3 0HA, UK

${ }^{8}$ Department of Physics, Hiroshima Univ., Higashi-Hiroshima, Hiroshima 739-8526

${ }^{9}$ Department of Physics, Aoyama Gakuin University, Sagamihara, Kanagawa 229-8558, Japan

${ }^{10}$ Department of Physics, Univ. of Tokyo, 7-3-1 Hongo, Bunkyo-ku, Tokyo 113-0033, Japan

${ }^{11}$ Physics Department, Kogakuin University 2665-1, Nakano-cho, Hachioji, Tokyo, 192-0015 email: aya@crab.riken.jp
}

\begin{abstract}
We present the results of six Suzaku observations of the recurrent black hole transient $4 \mathrm{U} 1630-472$ during its decline from its most recent outburst in 2006. All observations show the typical high/soft state spectral shape in the $2-50 \mathrm{keV}$ band, roughly described by an optically thick disk spectrum in the soft energy band plus a weak power-law tail.

The disk temperature decreases from $1.4 \mathrm{keV}$ to $1.2 \mathrm{keV}$ as the flux decreases by a factor 2 , consistent with a constant radius as expected for disk-dominated spectra. All the observations reveal significant absorption lines from highly ionized (H-like and He-like) iron $\mathrm{K} \alpha$ at $7.0 \mathrm{keV}$ and $6.7 \mathrm{keV}$.

The energies of these absorption lines suggest a blue shift with an outflow velocity of $\sim 1000 \mathrm{~km} \mathrm{~s}^{-1}$. The H-like iron $\mathrm{K} \alpha$ equivalent width remains approximately constant at $\sim 30 \mathrm{eV}$ over all the observations, while that of the He-like $\mathrm{K} \alpha$ line increases from $7 \mathrm{eV}$ to $20 \mathrm{eV}$. Thus the ionization state of the material decreases, as expected from the decline in flux.

The data constrain the velocity dispersion of the absorber to $200-2000 \mathrm{~km} \mathrm{~s}^{-1}$, and the size of the plasma as $\sim 10^{10} \mathrm{~cm}$ assuming a source distance of $10 \mathrm{kpc}$.
\end{abstract}

Keywords. Black hole physics - absorption lines 


\section{Introduction}

In recent years, a growing number of X-ray binaries have been found to exhibit absorption lines from highly ionized elements (e.g., Boirin et al. 2004 and references therein). These systems range from microquasars including GRO J1655-40 (e.g., Ueda et al. 1998; Yamaoka et al. 2001; Miller et al. 2006) to low-mass X-ray binaries including GX 13+1 (Ueda et al. 2004; Sidoli et al. 2002). These systems are viewed at high inclination angles. The absorption features are therefore thought to originate in material that is associated with and extends above the outer accretion disk. The recurrent black hole transient, 4U 1630-472, was observed 6 times with Suzaku (Mitsuda et al. 2006) from 2006 February 8 through March 23 during its most recent outburst as part of a program to study discrete spectral structures as a function of the changing accretion conditions (Kubota et al. 2006). We monitored the source during its decay from outburst, detecting significant absorption lines from highly-ionized iron throughout the observations.

\section{Continuum shape}

Figure 1 shows the 2-50 keV HXD/PIN (Takahashi et al. 2006; Kokubun et al. 2006) and XIS (Koyama et al. 2006) spectra of 4U 1630 - 472 divided by those of Crab spectra, which has an approximately power-law spectrum with $\Gamma=2.1$. This plot reveals several noticeable features in the broad band source spectra. The source spectra are characterized by a dominant soft thermal component in the XIS band, while the harder X-ray PIN data clearly show a weak power-law tail. The dominant soft component is generally observed from black hole binaries in the high/soft state (Tanaka \& Lewin 1995), and is believed to be emission from the optically thick standard accretion disk (Shakura \& Sunyaev 1973). In fact, this component is successfully reproduced with the multicolor disk model (DISKBB; Mitsuda et al. 1984; Makishima et al. 1986) modified by large interstellar absorption of $N_{\mathrm{H}} \sim 8 \times 10^{22} \mathrm{~cm}^{-2}$. For the two months observations, the disk inner temperature, $k T_{\text {in }}$, changed from $1.39 \mathrm{keV}$ to $1.18 \mathrm{keV}$ by keeping an apparent inner radius constant at $25 \mathrm{~km}$ for an assumed distance of $10 \mathrm{kpc}$ and inclination angle of $70^{\circ}$. Corresponding disk bolometric luminosity, $L_{\text {disk }}$, decreased from $2.8 \times 10^{38} \mathrm{erg} \mathrm{s}^{-1}$ to $1.6 \times 10^{38} \mathrm{erg} \mathrm{s}^{-1}$.

\section{Iron absorption lines}

The Crab ratios also reveal the presence of complex absorption structures in the iron $\mathrm{K}$ band. Figure $2 \mathrm{~b}$ shows an enlargement of the $6-9 \mathrm{keV}$ XIS spectra. The most obvious features are two narrow dips at $7.0 \mathrm{keV}$ and $6.8 \mathrm{keV}$, and weaker absorption structures are found at $7.8 \mathrm{keV}$ and $8.2 \mathrm{keV}$. We thus fit the $2-9 \mathrm{keV}$ data with the absorbed DISKBB model and 4 negative gaussians with fixed line width at $\sigma=10 \mathrm{eV}$. The time histories of the individual absorption-line equivalent widths and their energies are plotted in the left panel of figure 2. The time averaged center energies of the two strong absorption lines are estimated to be $6.987 \pm 0.005 \mathrm{keV}$ and $6.714 \pm 0.009 \mathrm{keV}$, which are consistent with H-like and He-like iron $\mathrm{K} \alpha$ suggesting a blue shift velocity of $900 \pm 200 \mathrm{~km} \mathrm{~s}^{-1}$ and $700 \pm 400 \mathrm{~km} \mathrm{~s}^{-1}$, respectively. The energies of two weaker absorption lines are almost consistent with $\mathrm{H}$-like and He-like iron $\mathrm{K} \beta$, though they can be contaminated by nickel $\mathrm{K} \alpha$. While the equivalent width of the H-like line was almost constant throughout the observations, that of the He-like line increased by a factor of two between the first and second observations, and then perhaps increased slightly more through the subsequent observations. These imply that the ionization state of the absorbing gas decreased over time, as expected from the declining source luminosity. 

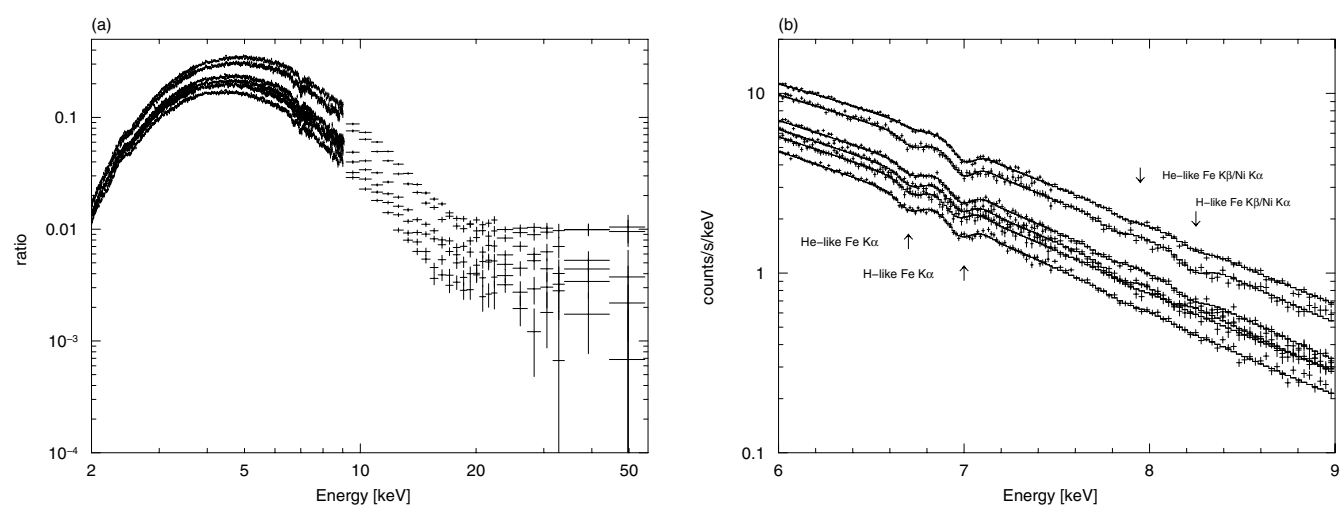

Figure 1. (a) The ratio of $4 \mathrm{U} 1630-472$ spectra to the Crab spectrum. Data are obtained with the XIS023 and the HXD PIN. (b) The XIS 6-9 keV spectra concentrating the absorption line structure. The data and the best fit models are shown.

We next fit the absorption lines with Voigt profile (e.g., Ueda et al. 2004) to estimate the absorbing ion column density, $N_{\text {ion }}$, under assumed velocity dispersion, $b$. Although we cannot measure $b$ from line profiles, its upper and lower bounds may be obtained. The upper limit is set at $b \sim 2000 \mathrm{~km} \mathrm{~s}^{-1}$ by the upper limits on the line width, $\sigma \sim 30-40 \mathrm{eV}$ (§ 3.2). The lower limit is estimated to be $\sim 200 \mathrm{~km} \mathrm{~s}^{-1}$ by comparing the curve-of-growth between $\mathrm{K} \alpha$ and $\mathrm{K} \beta$. Here, the ion column density estimated from the equivalent width of $\mathrm{K} \alpha$ with low velocity dispersion of $<200 \mathrm{~km} \mathrm{~s}^{-1}$ requires too high equivalent width for the $\mathrm{K} \beta$ of the same ion. The top three panels of figure 2(right) show the time histories of the ion column, the ratio of the He-like to H-like ions, and estimated blue shift, $z$, assuming $b=500 \mathrm{~km} \mathrm{~s}^{-1}$ which is based on the (marginally) resolved line width in the Chandra HETGS data of GX $13+1$ Ueda et al. (2004). The column density of the H-like iron, $N_{\mathrm{Fe} \text { XXVI }}$, is almost constant at $\sim 1 \times 10^{18} \mathrm{~cm}^{-2}$ while that of the He-like iron, $N_{\text {Fe XxV }}$, increases significantly from the first to the second observation. The weighted average blue shift is about $z=(3.3 \pm 0.7) \times 10^{-3}$, corresponding to an outflow velocity of $1000 \pm 200 \mathrm{~km} \mathrm{~s}^{-1}$.

\section{Physical parameters of the absorber}

Through the analyses, we found that iron is always predominantly $\mathrm{H}$-like, requiring that the ionization parameter, $\xi=L / n r^{2}$ (where $n$ and $r$ are the number density and the distance of the absorbing material from the illuminating source), be very high. In this section, we use the XSTAR photoionization code (version 2.1kn5; Kallman \& Bautista 2001) to calculate the ionization balance of the line-producing material, to convert the ion column densities into physical parameters including total column density, number density, and location, for each observation. We calculate the relative ion populations under illumination by a DISKBB spectrum, and use this to predict a theoretical ratio of $N_{\mathrm{Fe}} \mathrm{XXV}$ to $N_{\mathrm{Fe} \text { XXVI }}$. The observed ratio from the data then enables us to estimate the $\xi$-parameter, and so convert the observed ion column densities of $\mathrm{H}$-like and He-like irons into a total column density, $N_{\text {tot }}$.

The inferred time histories of $\xi$ and $N_{\text {tot }}$ are also shown in the right panel of figure 2 assuming $b=500 \mathrm{~km} \mathrm{~s}^{-1}$, together with upper and lower bounds corresponding to $b=$ 200 and $2000 \mathrm{~km} \mathrm{~s}^{-1}$. Assuming that $b$ stays constant at $500 \mathrm{~km} \mathrm{~s}^{-1}$ then there is a marginal decrease in $N_{\text {tot }}$ as the luminosity decreases, from $\sim 1 \times 10^{23} \mathrm{~cm}^{-2}$ in the first observation to $\sim 7 \times 10^{22} \mathrm{~cm}^{-2}$ in the final dataset, as well as a marginal decrease in $\xi$ 

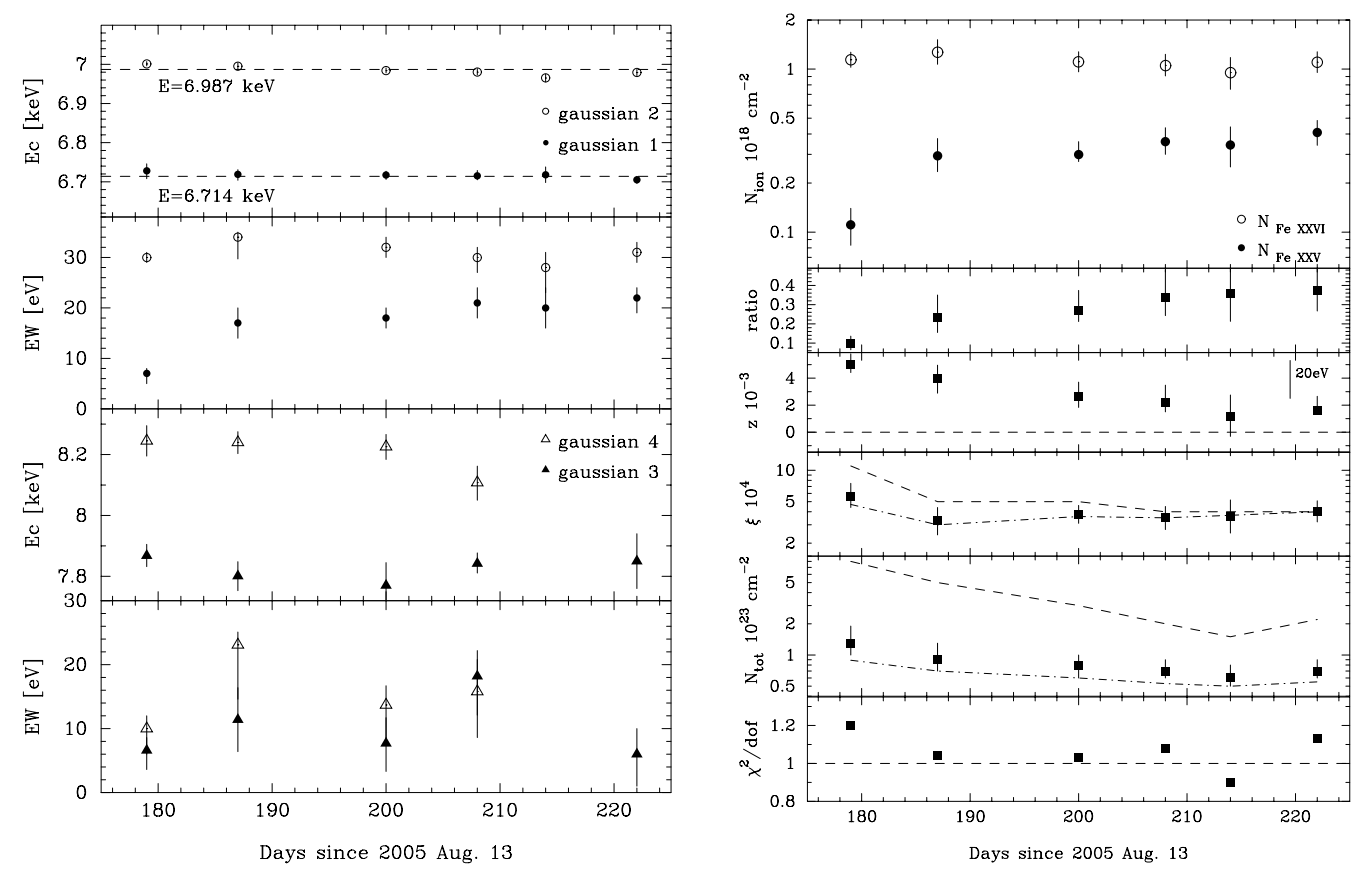

Figure 2. Time histories of absorption line parameters. Left: Based on the negative gaussian fit in the range of $6-10 \mathrm{keV}$. The first two panels show line center energies and equivalent width of the two strongest lines. The bottom two panels show those of weaker absorption features. Right: Based on the Voigt profile for $b=500 \mathrm{~km} \mathrm{~s}^{-1}$. Based on the ratio, $\xi$-parameter and $N_{\text {tot }}$ are shown in the next two panels. The best estimation of $\xi$ and $N_{\text {tot }}$ for $b=200 \mathrm{~km} \mathrm{~s}^{-1}$ (dashed line) and $2000 \mathrm{~km} \mathrm{~s}^{-1}$ (dash-dot line) are also shown in the same panels.

from $\sim 6 \times 10^{4}$ to $\sim 4 \times 10^{4}$. We can roughly estimate $n$ and $r$ of the absorbing plasma, by using the definitions of $N_{\text {tot }}=\bar{n} \Delta R$ with a mean number density $\bar{n}$ and a characteristic thickness of the absorber $\Delta R$.

Using $\xi=L / \bar{n} R^{2}$, the average distance of the absorber, $R$, is thus estimated as $R \sim$ $3 \times 10^{10} \cdot(\Delta R / R) \cdot D_{10}{ }^{2}$ and $\sim 4 \times 10^{10} \cdot(\Delta R / R) \cdot D_{10}{ }^{2} \mathrm{~cm}$, for the first and the last observations, respectively. The densities are also estimated as $\bar{n} \sim 5 \times 10^{12} \cdot(\Delta R / R)^{-1}$. $D_{10}{ }^{-2}$ and $\sim 2 \times 10^{12} \cdot(\Delta R / R)^{-1} \cdot D_{10}{ }^{-2}$ atoms $\mathrm{cm}^{-3}$. Assuming $\Delta R / R \sim 1$ by following the previous research, the values of $R$ and $\bar{n}$ are estimated to be on the order of $R \sim$ $10^{10} \cdot D_{10}{ }^{2} \mathrm{~cm}$ and $\bar{n} \sim 10^{12} \cdot D_{10}{ }^{-2}$ atoms $\mathrm{cm}^{-3}$.

These parameters are almost consistent with those obtained in other binary systems (e.g., Ueda et al. 1998; Kotani et al. 2000). This source joins the growing number of galactic binaries with such absorption, showing that winds are probably a generic feature of bright accretion disks.

\section{References}

Boirin L., Parmar A. N., Barret D., Paltani S., Grindlay J. E. 2004, A\&A, 418, 1061

Kallman T. R., Angelini L., Boroson B., \& Cottam J. 2003, ApJ, 583, 861

Kallman T. \& Bautista M. 2001, ApJS, 133, 221

Kubota A. et al. 2006 PASJ, in press

Kuulkers E., van der Klis M., Parmar A. N. 1997, ApJ, 474, L47

Kokubun M. et al. 2006, PASJ, in press

Kotani T. et al. 2000, ApJ, 539, 413 
Koyama K. et al. 2006, PASJ, in press

Makishima K. et al. 1986, ApJ, 308, 635

Miller J. M. et al. 2006, Nature, 441, 953

Mitsuda K. et al. 1984, PASJ, 36, 741

Mitsuda K. et al. 2006, PASJ, in press

Shakura N. I. \& Sunyaev R. A. 1973, A\&A, 24, 337

Sidoli L., Parmar A. N., Oosterbroek T., Lumb D. 2002, A\&A, 385, 940

Takahashi T. et al. 2006, PASJ, in press

Tanaka Y., Lewin W. H. G. 1995, in X-ray Binaries, eds. W. H. G. Lewin, J. van Paradijs, and W. P. J. van den Heuvel (Cambridge University Press, Cambridge), p. 126

Ueda Y. et al. 1998, ApJ, 492, 782

Ueda Y., Murakami H., Yamaoka K., Dotani T., Ebisawa K. 2004, ApJ, 609, 325

Yamaoka K. et al. 2001, PASJ, 53, 179

THOMAS MACCARONE: If the inclination angle of the system is such that most of the wind comes towards the observer, how would that change the estimate of the fraction of the accreted mass lost in the wind?

Aya Kubota: Outflow rate was estimated as $\rho v_{\text {wind }} 4 \pi r^{2} c_{f}$ ( $c_{f}$ is covering fraction of the wind). And the estimation assumes $c_{f}=0.1$, which corresponds to angles of $10-15^{\circ}$. So the estimated loss rate of $0.3 M_{\text {acc }}$ can be a secure lower limit for constant $\dot{\rho}$ being independent of $i$ for the covering fraction. But if the absorbing matter is mostly from a very small angle $\Delta i \ll 10^{\circ}$, it can make the outflow rate smaller. This consideration is model dependent. But roughly a factor three decrease in $\dot{M}_{\text {flow }}$ can be expected based on MHD. 


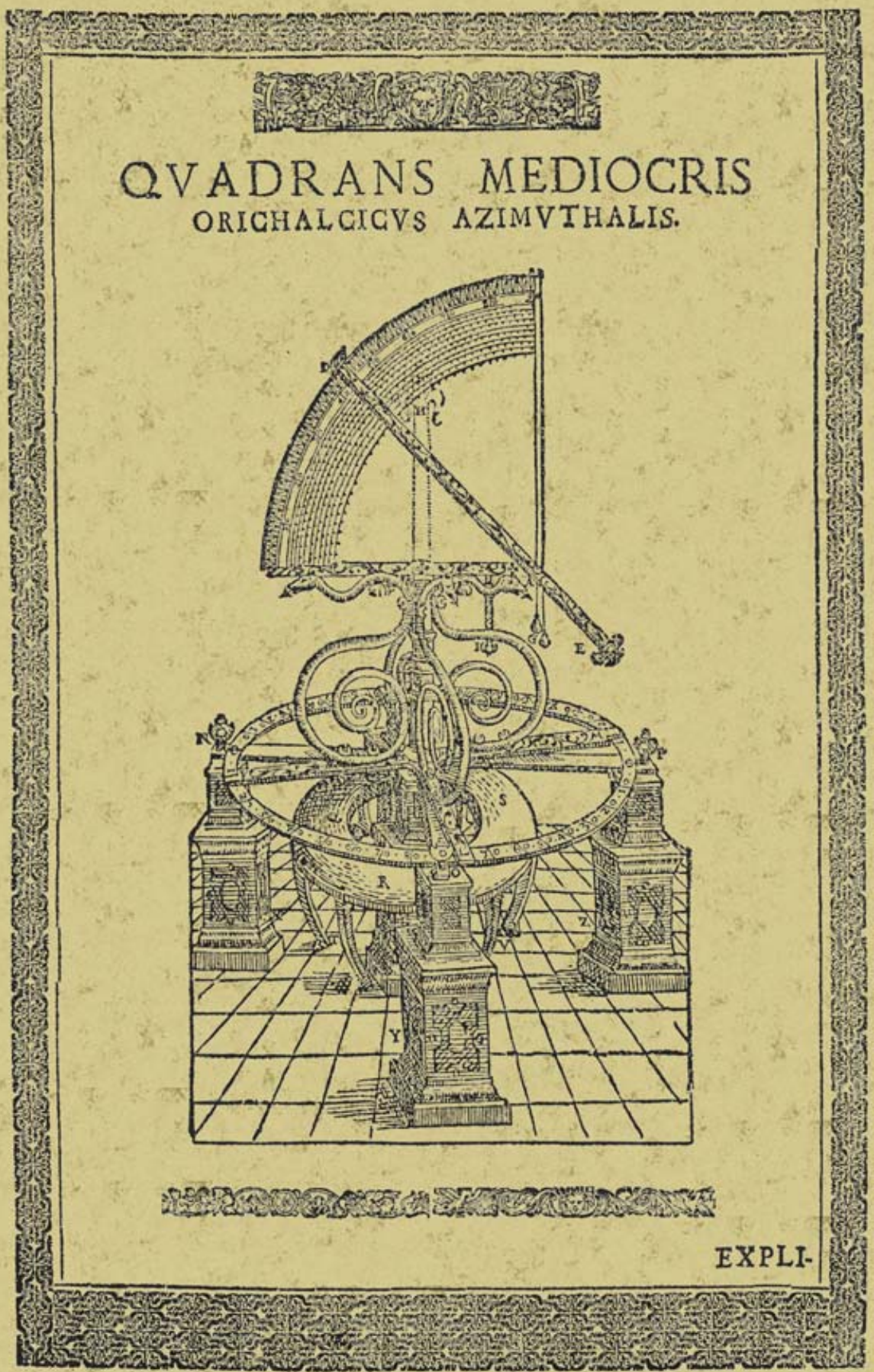

